\title{
$\mathfrak{J} \mathfrak{n} \mathfrak{h} \mathfrak{a} \mathfrak{l} \mathfrak{t}$
}

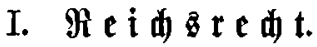

$9 x$.

1. Unbciugte Führung eines fremঠen Familiennamenక in der Firma; Er=

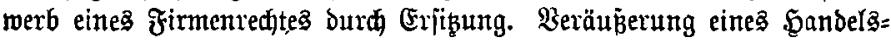
gcidäjtes . . . . . . . . . . . . . . . . . . . . . . 1

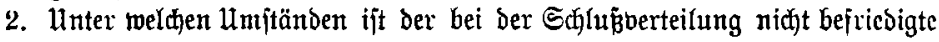

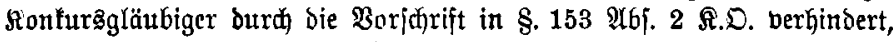

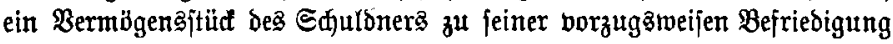
in $\mathfrak{A}$ nprud z̆ nebmen?.

3. Saftung ausgetretener Benoffen Ginfichtlia der Berbinblidteiten Der (Ge=

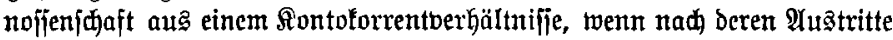
eine Saloveftitellung ftattgerunden hat und bie Salbojumme in neute ßed)ung vorgetragen $\mathrm{i} j \mathrm{t}$ ? . . . . . . . . . . . . . . . 11

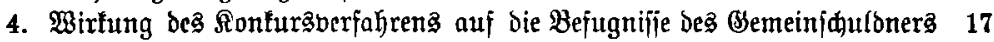

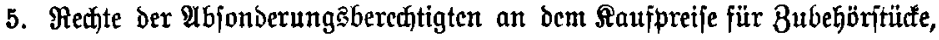

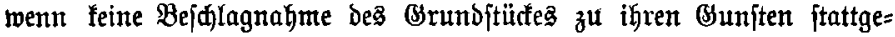
funden hat . . . . . . . . . . . . . . . . . . . 19

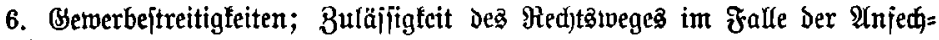

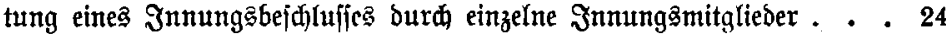

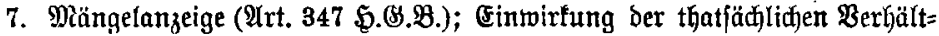
niffe auf die befondere Bejtaltung ber $\mathfrak{A n z e i g e ~ b e r ~}$ Pängel ber getauften Maren. . . . . . . . . . . . . . . . . . . . . . 27

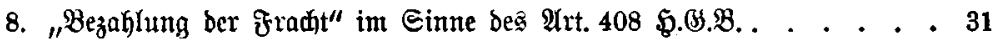

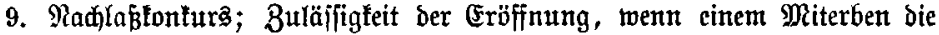

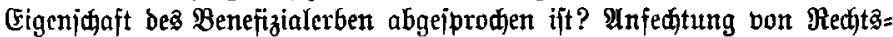
handlungen auf Srund dę \$. 23 R.D. . . . . . . . . . . .

10. Fingehung einer ftillen (Sejellidjaft Dura) eine Sommanditgeiellidhaft mit einem Dritten; Seilnahme bes ftillen Bejellinafters am (Sejelliajajts= getoinne . . . . . . . . . . . . . . . . . . . 
9x.

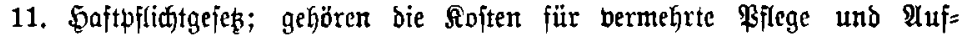
wartung beả Brrunglüaten zu ben Seilungafojten? Berpflid)tung zum

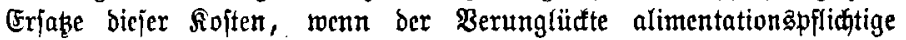
Ajcenbenten hat? . . . . . . . . . . . . . . . . .

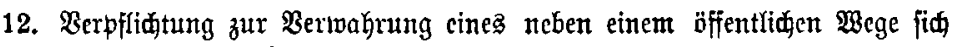
hinzichenden Grnbens. . . . . . . . . . . . . . . : . . 53

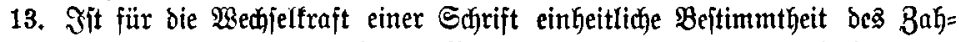
Iungsortes und ber zablenden Perjon mejentlid, Domizilmedjel . . . 56

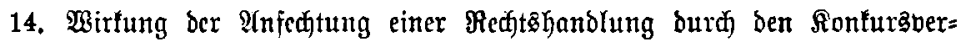

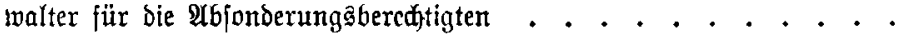

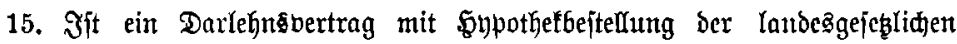

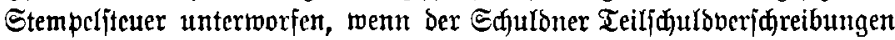

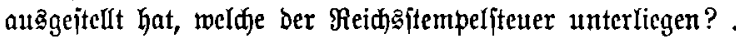

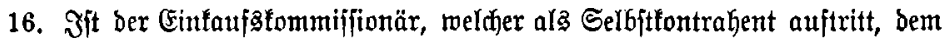
Fonmittenten gegenüber als wirflidjer Berfäufer antzujegen?. . . .

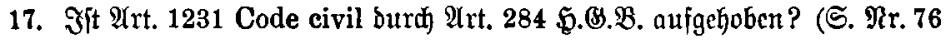

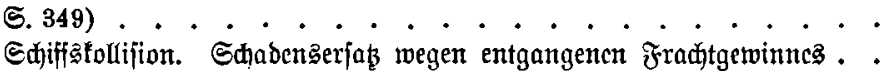

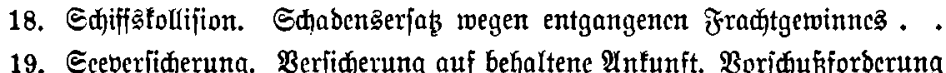
nad) ihrer paifibent Ecite als verfiderbares Jntereffe. . . . . . .

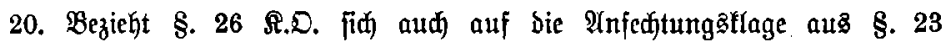
Biff. 2 イ.D.? . . . . . . . . . . . . . . . . . . . .

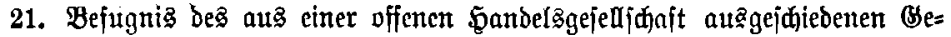
jellidnfters, bei ber Eimfiatnahme ber Sandersbüher ber Bejellfaft cinen Sadjoeritänbłgen zuzłżełent . . . . . . . . . . . .

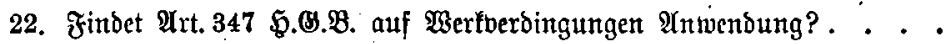

23. Ceeverficherung; Duarantänefipiten im Sinte ber Artt. 622. 757 S..(B.B. Bedeutung bcr Slaujel "nur für Seegefaht" . . . . . . . .

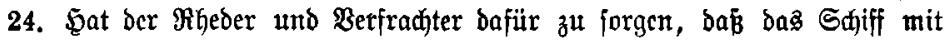

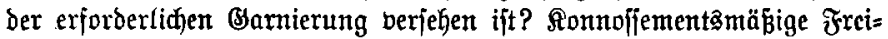

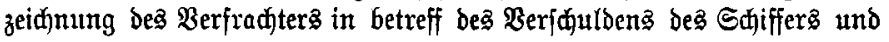
ber Shiffamantidajt . . . . . . . . . . . . . . . . .

25. Zur Beptimmung beas begriffliden Utnterjđiedes bon Fradtucrtrag und Sad): oder Dienitmiete. Beweislajt in betreff bes Beriduldens bei $F r=$ füllung von Berträgen . . . . . . . . . . . . . . . . .

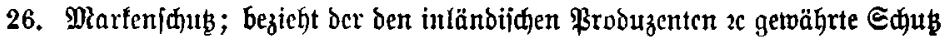

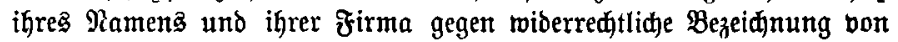

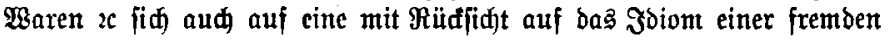
Epratje gemählte abweidente Sdjretbart des Ramens?? . . . . . .

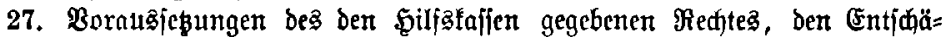
bigungäaniprud eineş bon ihnen Unteritüß̨ten gegen bie Unfallăberufäs genoffenjdajt geltend zu madfen . . . . . . . . . . . . . . 


\section{Gemeines Redt.}

$\Re i$

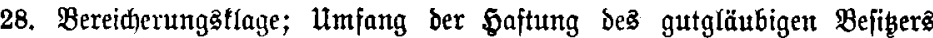
einer fremben beräuk̉erten Sađje bcm früfheren CFigentümer gegenüber megen Bercicherung? .

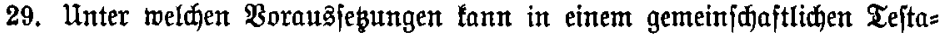

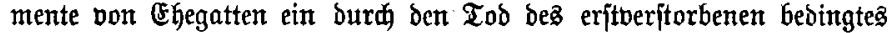

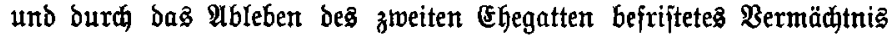

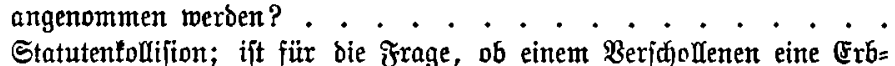

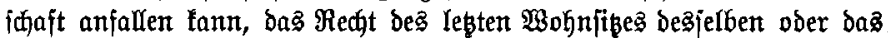

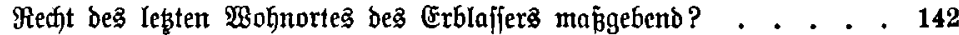

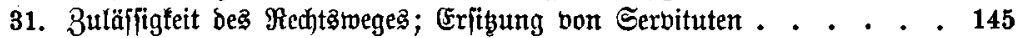

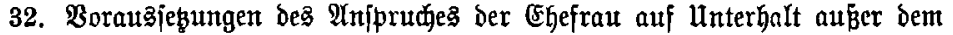
Sauje des Mantes . . . . . . . . . . . . . . . . . .

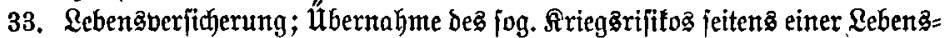
berjifjerungągejelfjaft auf Begenjeitigfeit . . . . . . . . .

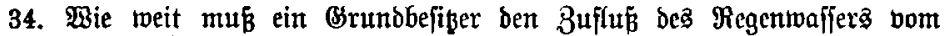
Radbargrundftüfe fid gefallen lajien? . . . . . . . . . .

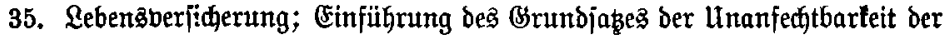
Bolicen; Wirkung für laufende Berfityerungen . . . . . . . . . 169

36. Rülfgabe ber dos an bie Frau zum Bwecfe ber Arimentation. Begrün=

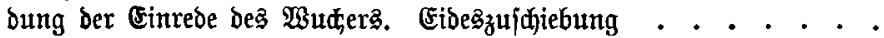

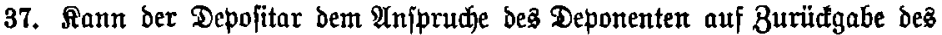

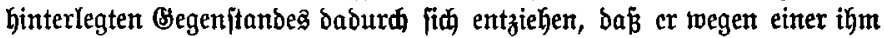
gegen ben (britten) (5igentümer besె (segenitandes zujtefenten Forberung einen $\mathfrak{A}$ refét auf ben lesteren erwirtt? . . . . . . . . . . . Selite

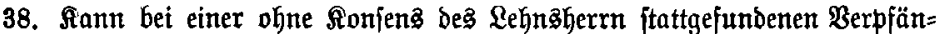

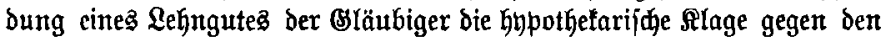
Berpfänocr und bie Rehnæafolger, weldje facta defuncti anzuerfennen

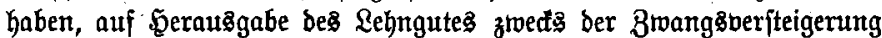
erheben? . . . . . . . . . . . . . . . . . 188

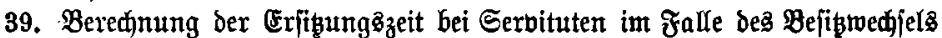
im bienenden Brundítüđe . . . . . . . . . . . . . . . 189

40. Slage auf Ungültigfeit ber Ełe megen mangelnber Jungfräulidfeit. . 192

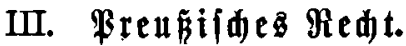

41. ErIaubte ßribatgejelljaft; notarielle Berfandlung über Benteralver=

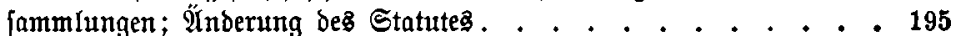

42. Regitimation burd nadfolgende che . . . . . . . . . . 204 

zum 巨d)eine erfolgt. . . . . . . . . . . . . . . . 207

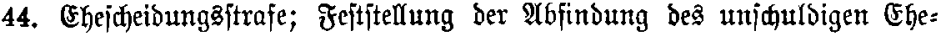
gatten auछె bem Bermögen dę̧ fđuldigen . . . . . . . . . . 211

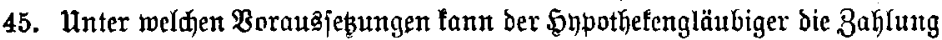

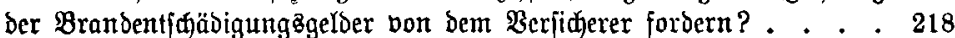

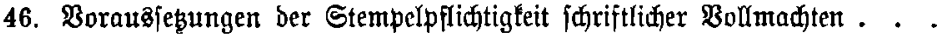

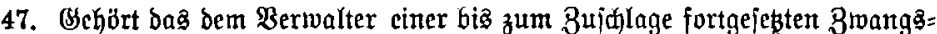
vermaltung gezahlte \$onorax zu Den aus dem Siaufgelde norweg zu be= riftigenden $\mathfrak{A} u \xi g$ gaben dę̧ betrctbenden Släubigers??. . . . . . .

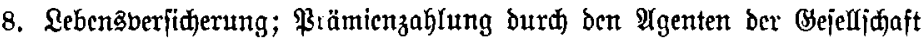

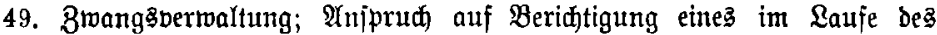
Berfahreng gegen bie 3roangâvermaltungamalje entitandenen $\mathfrak{A} n=$ \{prudes aus den Raufgeldern bor Don Forberungen ber Sybptheten= gläubiger . . . . . . . . . . . . . . . . . . . . . 237

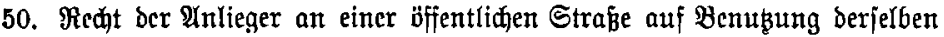

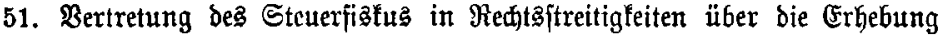
von Stempelabgaben.

52. Stemperjteuter für einen $\mathfrak{A}$ uãeinanderickungäbertrag unter Mitgliedern

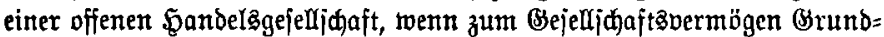
ftüđte gehören . . . . . . . . . . . . . . . . .

53. Steht aus einem gegen \$. 213 R.S. verftopenden Bejdäfte bem Beber

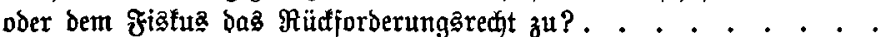

54. Sit eine bon einem Erblafjer eigenhänbig ge= unb unteridriebene Yek̨t= willige Berfügung, weldje twegen Mangels ber geridtliden form als

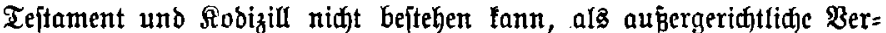
orbnung in ber Bejđränfung ber auşgejęten Bermäđtnifje biz zum zwanzigften Icile bes ఇađjlaffez gültig? . . . . . . . . . .

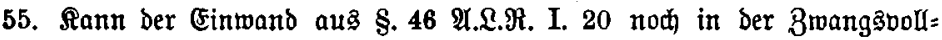

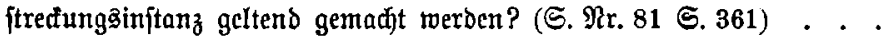

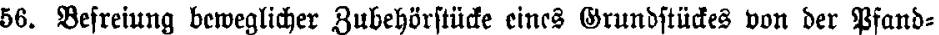
haftung burc Berfauf und räumlide Fortidaffung jeitens ocs (Figen=

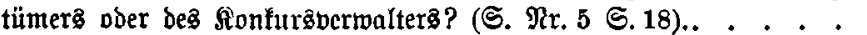

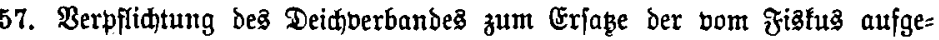

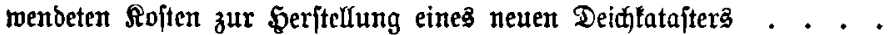

58. Bitalizienberrag; liegt cin joldjer vor, wenn ber û́bernehmer cinesิ Ber=

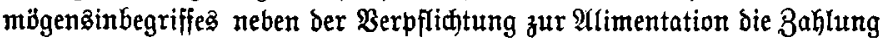
einer $\Re$ ente übernimmt? . . . . . . . . . . . . . .

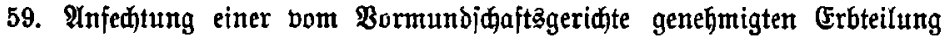

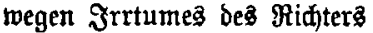


Mr.

60. Streit über ben Borrang zwijđen bem älteren ₹̧auftpfandgläubiger an

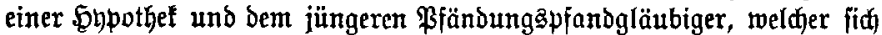

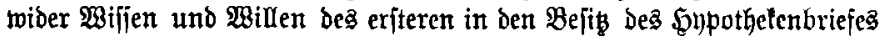
gejetht hat . . . . . . . . . . . . . . . . . .

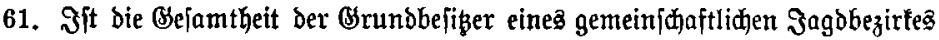

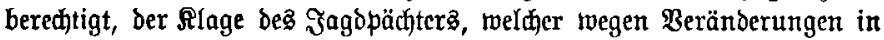

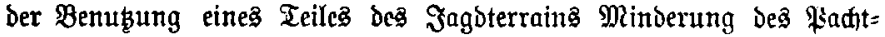

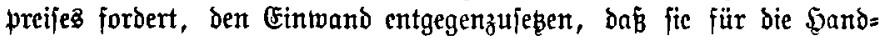
lungen der einzelnen Brumbbefizer nid)t hafte? (ङ. Nr. 77 S. 351) .

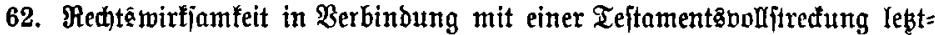
willig angeoroneter ßerjügungảbejđräntungen beą Bebađjten, an beren Iufredterbaltung fein Dritter intereffitert ijt. . . . . . . . . .

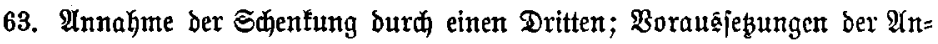

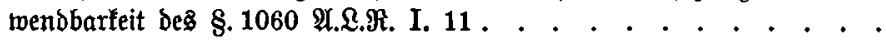

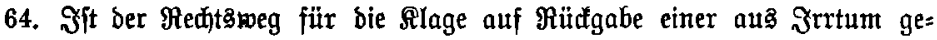

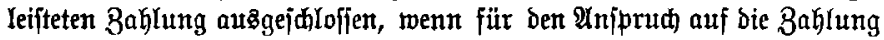

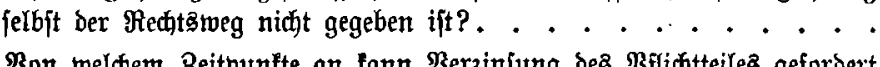

65. Bon weldem Beitpuntte an fann Berzinjung bea झflictteiles geforbert merben?...................... . . 307

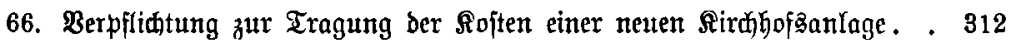

67. Mäłlergebühr . . . . . . . . . . . . . . . . . . . . 319

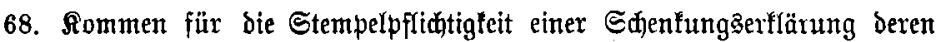
Annahme und Silagbarfeit in Betrad)t? . . . . . . . . . . 322

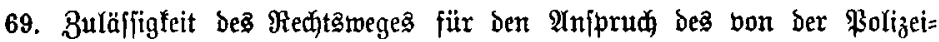

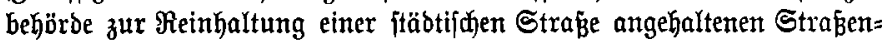
anliegers, baßs ber Stabtgemeinde als Eigentümerin der Straße bie Reinigungslaft obliege?

70. Einftelfung beż zur Beitreibung eines Stempelä anfängigen Bermal= tungazwangäberfahrens burch ba Beriđt, bei weldem bie Rlage auf

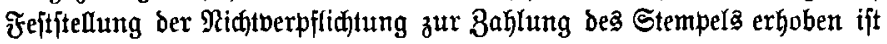

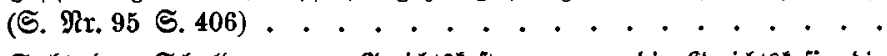

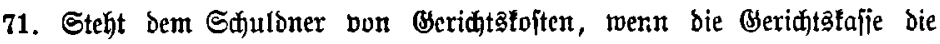

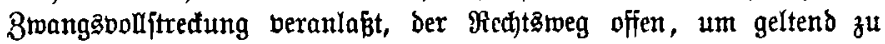

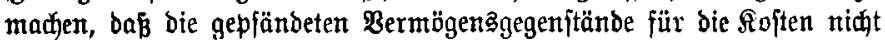
haften?

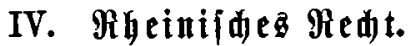

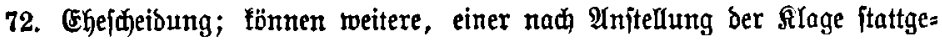

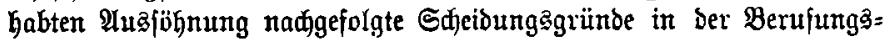
injtanz geltend gemađtt merben?. . . . . . . . . . . . 337 
Jithalt.

Rt. $\quad$ Eeite

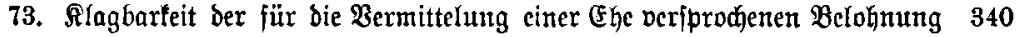

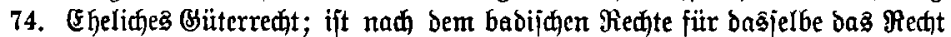

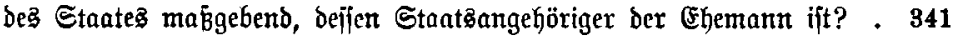

75. Silage auf IInterlaffung ber Fortieşung eincr unredten That. . . , 347

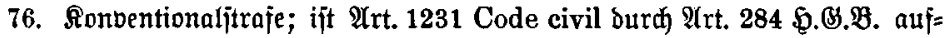
gefoben?....................... 349

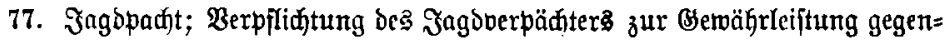

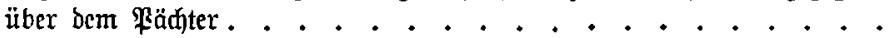

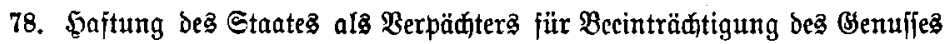

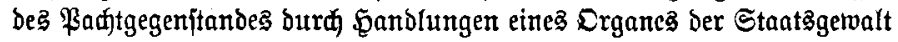

\section{Prozeñredt.}

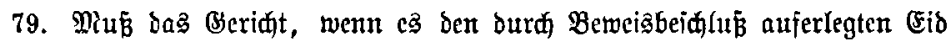

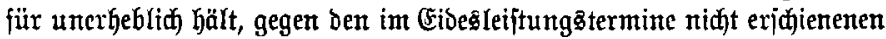

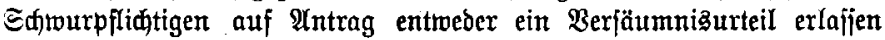
vber ben Antrag zurüftweijen? . . . . . . . . . . . . . .

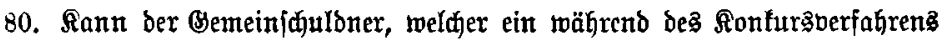
gegen ihn ergangenes $\mathfrak{u}$ rteil hat zujtellen lajien, bie Buläjijgfeit ber vom Begner eingelegten Berufung Deăhalb beftreiten, weil bie $8 \mathfrak{3}$ itellung un= gülttig jei? (S. Mir. 4 S. 17) . . . . . . . . . . . . . . .

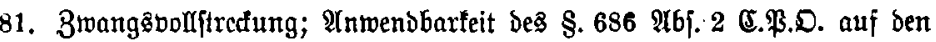

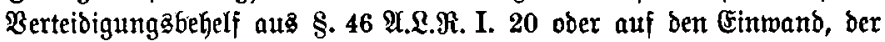

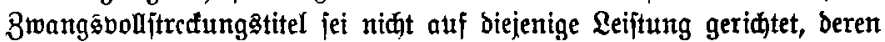
Erzivingung beantragt worbon? .

82. Beitreibung ber im Strafberfahren erfannten Beld ftrafen und ber ßoften bes @trafuerfahrenz; Rechtahillfe. . . . . . . . . . . . . . 364

83. Strcitmert bet ber Silage auf Feitfellung einer Bürgifaftäjuldb. . . 366

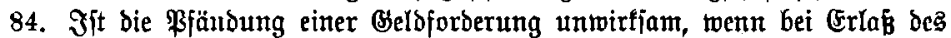

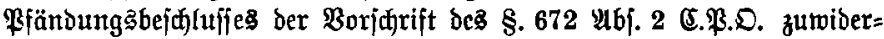
gehandelt war? . . . . . . . . . . . . . . . .

85. Bebeutung ber in ben Fällen ber Finlegung bes (Finjpruđes oder eineş

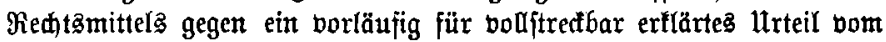

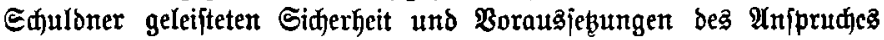
auf beren $\Re$ üdgabe.

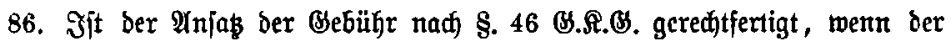

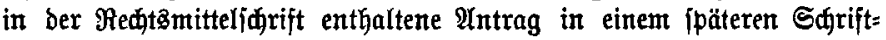
jabe eingejdränft $i f t$ ? . . . . . . . . . . . . . . .

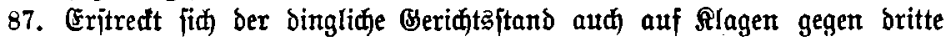

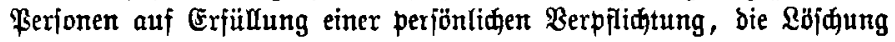
zu berirfen? . . . . . . . . . . . . . . . . . . . 
Rr.

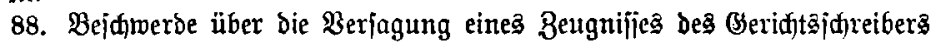

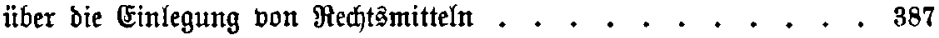

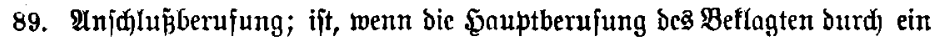

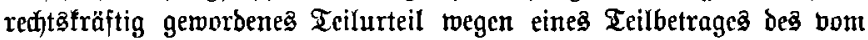

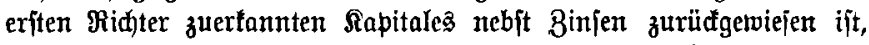
bei ber Berhandlung Der Berufung über Den Reftbetrag jenes Bapitalez

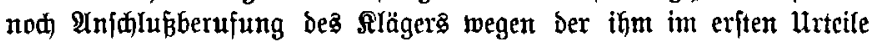
aberfannten Binjen zuläffig? (S. Nr. 65 S. 307) . . . . . . .

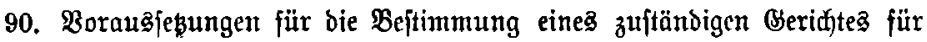
eine STage, weldye auf mehrere, in ben Bezirfen beridjiedener Breridjte belegene, unbelveglidje Sadjen geridjet ift . . . . . . . . .

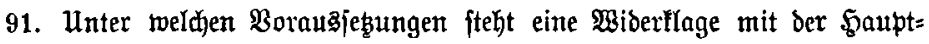
Hlage im 3 ufammentange $(\$ .33$ ( $\$ . \$ .0$.$) ? . . . . . . . . .$

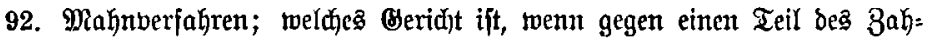

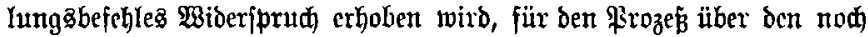
jtreitigen Teil fad)Yid zuftändig? . . . . . . . . . . . . .

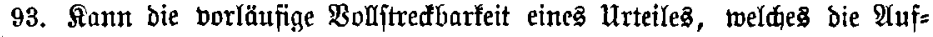
hebung eines Arreftes ausfpriat, in ber Berufungsinjtanz bor ber münd=

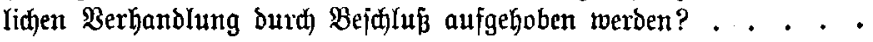

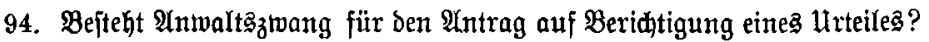
401

95. Cinjtellung beş zur Beitreibung eines Stempels angeoroneten $\mathfrak{B e r w a l}=$

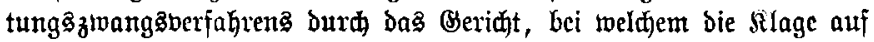
Feftitellung ber Nidtberpflidtung zux Bahlung des Stempels erhoben iit

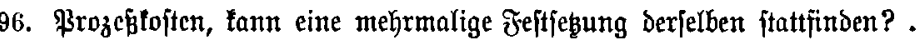

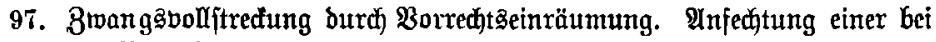

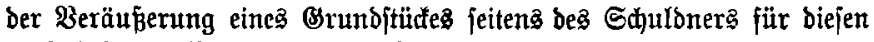

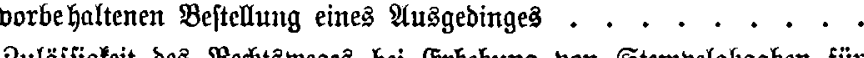

98. Buläifitgleit bes Rechtämeges bei Erhebung von Stempelabgaben für Afte ber freilvilligen Beridhtäbarfeit. Sompetenzfonflift . . . . .

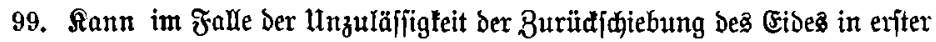
Injtanz ber Zurüałfiebende in ber Berulungsinjtanz neue Beweiz=

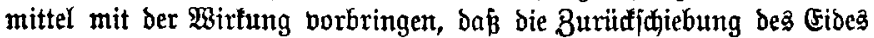

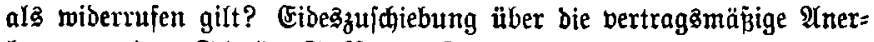
fennung einer Sthulb (S. Mr. 36 S. 177). . . . . . . . . .

100. Entmünbigungąberfahren; welđem Beamten ber Staatşantvaltiđaft ift bie Berufungajfdrift zuzuftellen. . . . . . . . . . . . .

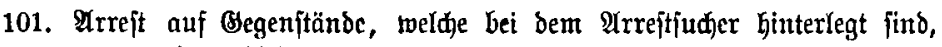
wegen eincr biejem gegen ben britten (Figentümer der finterlegten

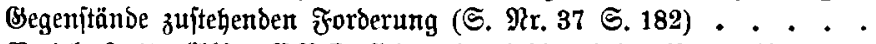

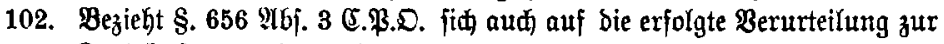

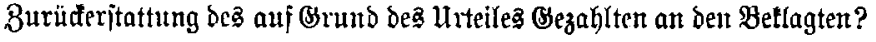


$\mathfrak{R r}$.

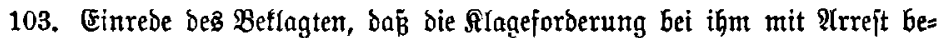
Iegt fei; ift jie ins 3mang

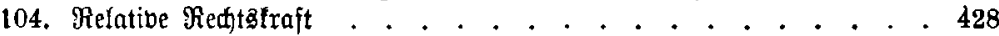

હafjregifter . . . . . . . . . . . . . . . . . . . . . . 434

Belebegregifter . . . . . . . . . . . . . . . . . . . . . 454

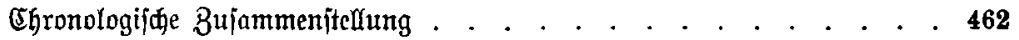

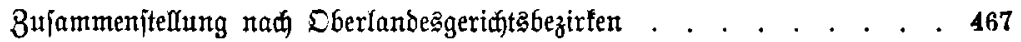

Beridtigungen . . . . . . . . . . . . . . . . . . . . . 468 